\title{
A Mesh-Free Compliant-Wall Fluid-Structure Interaction Model
}

 \\ ${ }^{a}$ Fluid Dynamics Research Group, Curtin University, \\ GPO Box U1987, Perth, Western Australia, 6845 \\ Email: j.kapor@curtin.edu.au
}

\begin{abstract}
This paper presents the development of a numerical algorithm for the simulation of closely coupled fluid-structure interaction (FSI) systems. The particular FSI system investigated in this work involves a high-Reynolds number flow over a single-sided compliant wall section between rigid baffles upstream and downstream. This system is a fundamental analogue of many complex FSI systems found in nature ranging from biomedical applications to drag-reduction using compliant coatings. The present study compares the efficacy of various numerical techniques to resolve the fully-coupled, non-linear FSI dynamics. Of particular interest is the resolution of coupled dynamics at fluid-structure density ratios of approximately unity where typical segmented solution techniques tend to have difficulties. Numerical techniques for resolving these tightly coupled dynamics are crucial to the development of generalized workable grid-free computational methods based on boundary-element and discrete vortex formulations.

The flow in this study is represented numerically as an ideal or potential axial flow, however it is important to note that the numerical schemes developed are equally applicable to rotational and viscous flow fields. The flow over the non-linear deforming surface is handled by a boundary-element method formulation of the Laplace equation. The structural dynamics are represented numerically by a finite-difference formulation of the Euler-Bernoulli beam equation. Various algorithms for the coupling of the fluid and structure equations will be tested for their numerical efficiency, stability and overall accuracy. The particular algorithms of note involve the semi-implicit, the linearised fluid inertia and the fully-implicit coupling methods.
\end{abstract}

The compliant-wall is modelled using a one-dimensional (1D), non-linear, Euler-Bernoulli beam model, with the non-linearity captured through an induced tension term. We look at the transient response obtained from the initial value problem, with the solution obtained numerically through an implicit time stepping scheme and the finite difference method (FDM). In all cases, the $\mathrm{O}\left(n^{2}\right)$ computational complexity that is typical with the numerical solution of a boundary-element formulation is mitigated through the use of a fast-multipole method (FMM) that reduces the complexity to $\mathrm{O}(n \log n)$. Thus, the numerics are handled in such a way that system matrices are not explicitly formed and thereby avoiding issues of associated memory storage.

The results validate well against previously published experimental and numerical work. They show that the semi-implicit method is an efficient computational technique for the solution of low density-ratio FSI problems, however it fails to achieve convergence at high density ratios. The fully-implicit coupling method achieved a good convergence and efficiency in the case of high density ratio models, however it's computational cost was higher than the semi-implicit method, but still lower than the coupling of the linearised fluid inertia term. Further work in this area will involve using these results to facilitate modelling fluid-structure systems that incorporate the dynamics of full viscous and rotational flow.

Keywords: boundary element method, compliant wall, fast multipole method, fluid-structure interaction, Newton-Krylov, matrix-free 


\section{INTRODUCTION AND BACKGROUND}

This paper presents the development and comparison of algorithms suitable for the numerical simulation of the Fluid-Structure Interaction (FSI) system illustrated by Fig. 1 in which an ideal axial flow interacts with a deforming flexible panel, or compliant wall. This system has a long history of investigation in aero/hydro-elasticity often being described as the problem of panel flutter because with increasing flow speed the panel undergoes divergence, or buckling, instability due to the fluid forcing that is replaced at higher flow speeds by violent and often destructive flutter instability. Theoretical studies have largely been based on linearised models by assuming low-amplitude disturbances of the wall while more complex fluid-flow models that include viscous effects require the assumption of a panel of infinite length in a normal-mode decomposition, boundary-value, formulation. A complete model of the fully coupled system will only be achieved using computational methods. Progress has been made in this regard; for example Lucey et al. [1997] simulated nonlinear dynamics using ideal flow while low-amplitude disturbances in viscous channel flow with a compliant insert have been simulated by Davies and Carpenter [1997] and for large amplitude deformations by Luo et al. [2008] using grid-based methods that are viable at relatively low Reynolds numbers.

The overall aim of the present work is to develop a versatile workable grid-free computational model for high Reynolds-number flows in the system using a combination of discrete-vortex method (DVM) for viscous boundary-layer effects in the flow, boundary-element method (BEM) for the irrotational component of the flow and finite-difference method (FDM) for the wall dynamics. As an essential step towards this aim, the specific thrust of the present paper is the development and assessment of stable, accurate and efficient time-stepping algorithms for the simulations. To facilitate this, we work with an ideal (infinite Reynolds number) flow but our design is framed by the requirement that the resulting algorithms can equally incorporate the DVM model of viscous effects and flow rotationality. In what follows, we first present an efficient scheme for the FDM solution of the wall dynamics and from that build the fully-coupled fluid-loaded system using the BEM. We then present three approaches to the time-stepping solution of the system's governing equation. In closing we comparatively summarise the merits and limitations of each providing recommendations of their utility particularly with respect to the fluid-to-solid density ratio of the system being studied.

\section{Wall Model}

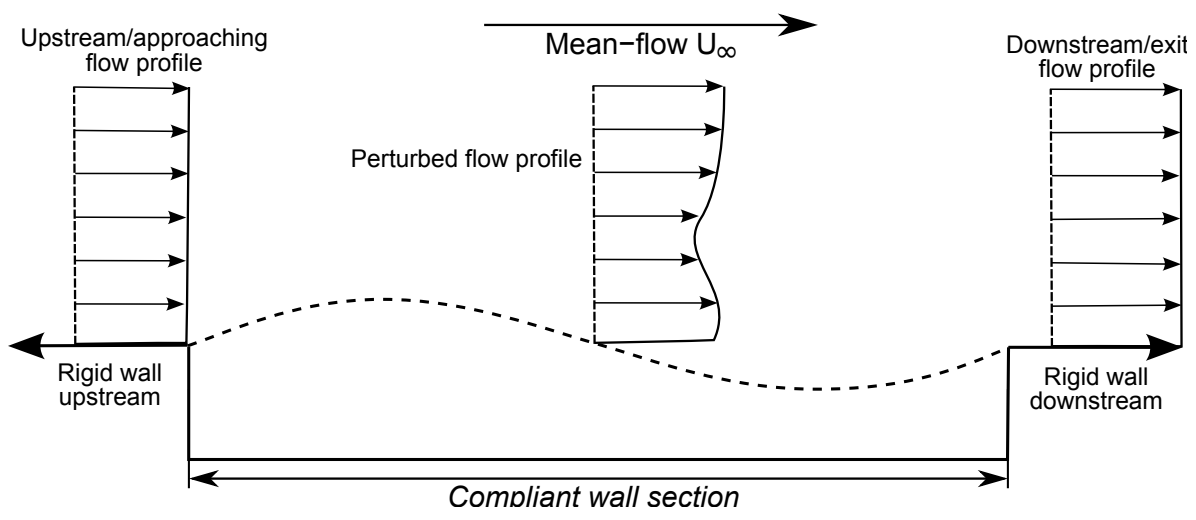

Figure 1. Schematic of the FSI model.

For the compliant-wall model, as shown in Fig. 1, we use the 1D, nonlinear, Euler-Bernoulli-Beam model which is presented in Lucey et al. [1997]. Nonlinearity is captured through an induced tension term that results from elongation caused by curvature in the wall. The governing differential equation for 
the vertical wall motion, $\eta=\eta(x, t)$, is given by,

$$
\rho h \frac{\partial^{2} \eta}{\partial t}+B \frac{\partial^{4} \eta}{\partial x^{4}}-T_{I}\left(\frac{\partial \eta}{\partial x}\right) \frac{\partial^{2} \eta}{\partial x^{2}}=F(x, t),
$$

where $\rho$ is the wall density, $h$ is the thickness and $F(x, t)$ is the force applied at the wall's surface. In addition, $B$ denotes the flexural rigidity of the plate and $T_{I}$ is the induced tension coefficient given by,

$$
B=\frac{E h^{3}}{12\left(1-v_{p}^{2}\right)} \quad, \quad T_{I}=\frac{E h}{L\left(1-v_{p}^{2}\right)} \int_{0}^{L}\left(\sqrt{1-\left(\frac{\partial \eta}{\partial x}\right)^{2}}-1\right) \mathrm{d} x
$$

where $v_{p}$ refers to Poisson's ratio, $E$ is the elastic modulus and $L$ is the length of the undeformed wall.

A solution to the system is achieved numerically by discretising the continuous wall into $N$ nodes of mass, uniformly spaced by $\Delta x$ in the horizontal direction. By keeping the separation of the nodes uniform we can apply second-order central-difference approximations [Trefethen, 1996] to all spatial differentials. End-joints are chosen to be hinged and implemented numerically through the addition of phantom nodes at the wall ends.

In this work, we look at the transient response obtained from the initial value problem where time integration is achieved through a second-order, implicit, trapezoidal stepping method. The use of an explicit time-stepping method has been avoided as the numerical stiffness of the equations of motion results in the convergence rate and stability of the problem being highly dependent on wall discretisation and time-step sizes. An increase in spatial discretisation requires a much larger increase in time discretisation to ensure numerical stability, well beyond the necessity for solution accuracy. By using an implicit method, relative time-step sizes no longer impact the numerical stability of the solution.

\subsection{Implicit solution method}

The trade-off with using an implicit method is that to obtain a solution we must find the roots of the system equations in the form $f(\ddot{\eta})=0$. Where the system equations are analytically defined before hand, methods such as the Newton-Raphson (NR) approach can be used, however the inclusion of nonlinear tension makes obtaining analytical forms of the derivative (or Jacobian, $[J]$, for systems of equations) impossible. While numerical approximation and inversion of the Jacobian matrix is possible through finite-difference and Gaussian-elimination, with increasing levels of wall discretisation and the Jacobian's square shape $(n \times n)$, these operations becomes prohibitively expensive on the order of $O\left(n^{2}\right)$ and $O\left(n^{3}\right)$ respectively.

A better alternative for large systems of nonlinear equations is the Newton-Krylov (NK) method [Knoll and Keyes, 2004]. This is based upon the use of a finite-difference approximation (typically first-order) to the Jacobian-vector product used in the NR. The advantage of this formulation is that one can use a Krylov subspace method, such as the Generalised Mean Residual method (GMRES), to iteratively determine the vector product. This method is suited to large systems of nonlinear equations because only system function $(f)$ evaluations are required which allows the whole scheme to be conducted in a matrix-free manner.

However, for good performance of any iterative Krylov method, the system of equations must be well conditioned. To achieve this we must develop a pre-conditioner that can be used in the Krylov calculations to provide a good approximation for the inverse of the Jacobian matrix. Here the problem lies in the fact that; i) the Jacobian does not explicitly exist, and obtaining it would be numerically expensive $\left(O\left(n^{2}\right)\right)$, ii) even with a fully defined Jacobian matrix, its direct inversion is numerically expensive $\left(O\left(n^{3}\right)\right)$, and iii) the preconditioner must be able to reduce the total number of Kyrlov and Newton iterations.

The solution proposed in this paper is a static pre-conditioner that is obtained by generating an approximate Jacobian matrix through finite differences. This is applied on a sparsifying stencil that ignores the influence of elements outside of a 2 element radius, which is suitable due to the diagonal dominance of the Jacobian. The end result is a sparse, diagonal banded matrix that approximates the Jacobian; however as opposed to direct inversion, we use the Incomplete-LU Decomposition method to provide an approximate inverse to this matrix. 


\section{FLUID-STRUCTURE INTERACTION}

The Boundary Element Method (BEM) is a technique that has been applied to the compliant-wall potential-flow FSI problem before in Lucey et al. [1997] and for nonlinear complaint deformations in Pitman [2007] with good success. We use this method to achieve the fluid-wall coupling by utilising source/sink panels that follow the wall motion and are located between the mass nodes. While the background of the BEM is not discussed here, one should see Katz and Plotkin [1991] for the general method and Kapor et al. [2010] for examples of applications to the present configuration.

Summarised, the key features that make the BEM ideal for the present problem are; i) it's ability to model the entire flow field using a surface integral method, ii) it's mesh-free nature allows easy deformation of the fluid-wall boundary, and iii) pressure forces at the fluid-wall boundary can be calculated with relative ease.

Where the fluid-flow perturbs under the wall motion it also offers a reactionary response back onto the wall through its corresponding change in pressure. To determine the pressure in the flow field one can apply the unsteady Bernoulli equation for potential-flow which can be coupled to the wall through the forcing term, $F(x, t)$, in Equation 1 as,

$$
F(x, t)=-\Delta p(\ddot{\eta}, \dot{\eta}, \eta)=\rho_{f}\left(-\frac{U_{\infty}^{2}}{2}+\frac{\partial \phi}{\partial t}+\frac{U^{2}+V^{2}}{2}\right),
$$

where $\rho_{f}$ is the fluid density, $U_{\infty}$ is the undisturbed velocity seen in Fig. 1 and the time differential is obtained by a standard, second-order backwards difference approximation. $\phi, U$ and $V$ are the perturbation velocity potential, horizontal and vertical velocities respectively in the fluid at position $x$ and are obtained from the BEM at the node midpoints using,

$$
[\phi]=\left[I_{\phi}\right][\sigma] \quad, \quad[U]=\left[I_{U}\right][\sigma] \quad, \quad[V]=\left[I_{V}\right][\sigma] .
$$

Here $[\sigma]$ denotes the vector of source/sink panel strengths of size $N \times 1$ and $\left[I_{*}\right]$ denotes the corresponding influence matrix of size $N \times N$. The influence matrices are purely a function of panel angles and positions which change in time. As it is undesirable to form and manipulate large square matrices, due to their $O\left(n^{2}\right)$ complexity, the Fast Multipole Method (FMM) [Greengard and Rokhlin, 1987] is used to perform the vector product in an efficient $O(n \log n)$ scheme, previously presented in Kapor et al. [2010].

While the FMM may offer a fast matrix-vector product, this is only possible once the panel strength vector $([\sigma])$ is known. To determine the strengths we solve the set of equations that enforce the no-normal velocity at the centre of each panel,

$$
\begin{aligned}
0 & =\left[I_{v}\right][\sigma]+\left[V_{\text {norm }}\right] \\
{[\sigma] } & =\left[I_{v}\right]^{-1}\left(\left[-U_{\infty} \sin \theta_{p}-\frac{\partial \eta_{p}}{\partial t} \cos \theta_{p}\right]\right),
\end{aligned}
$$

where $I_{v}$ is the square influence matrix made by the panel-normal component of the velocity. From this relationship the changing wall motion impacts the flow-field in three ways via; i) the position affecting the relative angle of the panel to the mean-flow direction, ii) the curvature in the panel-normal matrix, and iii) directly through the coupled wall velocity. As opposed to actually solving for the inverse of the normal-influence matrix, we again use the GMRES for greater efficiency and to abide by the matrix-free theme of the model. With the GMRES we also utilise the FMM for the matrix-vector product $\left(\left[I_{v}\right][\sigma]\right)$ to avoid the costs of the direct $O\left(n^{2}\right)$ calculation.

Rewriting the full potential-flow FSI system of equations by substituting Equation 3 into Equation 1 we have,

$$
\rho h \frac{\partial^{2} \eta}{\partial t^{2}}+B \frac{\partial^{4} \eta}{\partial x^{4}}-T_{I}\left(\frac{\partial \eta}{\partial x}\right) \frac{\partial^{2} \eta}{\partial x^{2}}=\rho_{f}\left(-\frac{U_{\infty}^{2}}{2}+\frac{\partial \phi}{\partial t}+\frac{U^{2}+V^{2}}{2}\right) .
$$

\subsection{Solution Method}

The three components on the right hand side involve full $(n \times n)$ matrices which causes the evaluation of the right hand side of Eqn. 7 to be very expensive. Dynamic calculation of these terms should be avoided 
in the implicit NK calculations as this would add an $O\left(n^{2}\right)$ component to the function $(f)$ calls, whereas without it, all components are of order $O(n)$. An alternative approach to a fully implicit coupling is to use a semi-implicit coupling of the forcing function by,

$$
\left[\rho h \frac{\partial^{2} \eta}{\partial t^{2}}+B \frac{\partial^{4} \eta}{\partial x^{4}}-T_{I}\left(\frac{\partial \eta}{\partial x}\right) \frac{\partial^{2} \eta}{\partial x^{2}}+\rho_{f} \frac{U_{\infty}^{2}}{2}-P^{*}\right]_{t}=\left[\rho_{f}\left(\frac{\partial \phi}{\partial t}+\frac{U^{2}+V^{2}}{2}\right)-P^{*}\right]_{t^{*}} .
$$

Here we iterate between the implicit solution on the left hand side and semi-implicit correction on the right hand side until $t^{*}=t$. An additional $P^{*}$ term has been included on both sides of the equation to act as an optional conditioner that influences the overall complexity of the implicit coupling. The choice of this term is complicated and must be discussed further.

No Conditioning. If we first provide no semi-implicit conditioning we have,

$$
P^{*}=P_{1}=0 .
$$

This provides the simplest form of coupling with the NK on the left hand side solving the same as it would in vacuo, with the full $O\left(n^{2}\right)$ fluid calculations now restricted to each semi-implicit iteration.

Linearised Inertia Conditioning. By looking at the order of the terms on both sides of the system equation it is seen that a large influence in the fluid-wall coupling is the unsteady pressure term, $\frac{\partial \phi_{t}}{\partial t}$. By performing a linear analysis [Pitman, 2007] we can see that for 'heavy' fluids the linearised fluid inertia is the component with the greatest influence on wall acceleration. It is beneficial to shift this term to the left hand side of the equation to be solved implicitly and then allowing the nonlinear components to be applied semi-implicitely. This is achieved by setting,

$$
P^{*}=P_{2}=2 \rho_{f}\left[I_{\phi}\right]\left[\frac{\partial \eta_{p}}{\partial t}\right] .
$$

The addition of this coupling allows the semi-implicit pressure on the right hand side to be more resilient to changes in the wall acceleration. While this results in faster convergence and higher numerical stability, it does come at a cost, with evaluations of $\left[I_{\phi}\right]$ being required for every call of $f$. The change of $f$ also requires modification of the Jacobian preconditioner, but due to the diagonal nature of $P_{2}$ this is a simple inclusion in the existing method.

Full Potential-Flow Implicit Coupling. The third option is to utilise the fully coupled, nonlinear, potential-flow as the conditioner by,

$$
P^{*}=P_{3}=\rho_{f}\left(\frac{\partial\left(\left[I_{\phi}\right][\sigma]\right)}{\partial t}+\frac{\left(\left[I_{U}\right][\sigma]\right)^{2}+\left(\left[I_{V}\right][\sigma]\right)^{2}}{2}\right) .
$$

Here the time differential is solved as previously with a second-order backward difference method and the strength $(\sigma)$ is solved via GMRES and the FMM to satisfy the no-flux boundary condition. The advantage of this method is that the right-hand terms cancel, leaving the nonlinear system fully coupled to the fluid implicitly, requiring no semi-implicit iterations. The disadvantage is that the Krylov iterations are now even more complicated over the linearised version, requiring an additional fluid field calculation (FMM) and an inverse matrix-vector product (GMRES). In addition, the inverse Jacobian preconditioner to the NK is now dense, however as the fluid matrices are still diagonally dominant it responds well to the existing sparsification scheme.

\section{Illustrative Numerical Results}

For validation purposes, we provide an illustrative example of the FSI of air flow over an elastic rubber type wall $\left(\rho_{f} / \rho=1.17 \times 10^{-3}\right)$, with the flow speed set to ensure energy-stable, nonlinear, divergence instability $(\Lambda=202)$ [Lucey et al., 1997]. Fig. 2(a) shows snapshots of the wall positions through time using the $P_{1}$ scheme, with snapshots taken every $1.6 \times 10^{-3}$ s over a single oscillation. Fig. $2(\mathrm{~b})$ shows the vertical position of the central wall node through time for all three coupling methods, clearly illustrating good agreement and the nonlinear oscillatory behaviour. For this case, conditioning scheme $P_{1}$ computed 


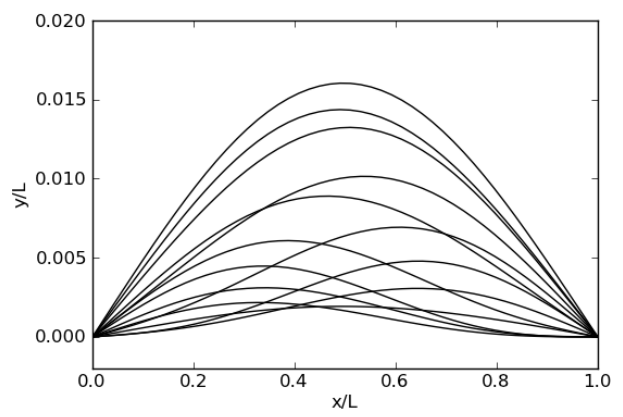

(a) Wall position transient snapshots.

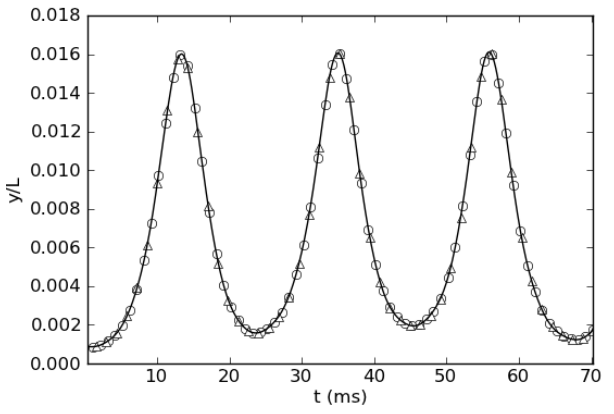

(b) Wall midpoint trace for $P_{1}$ (line), $P_{2}$ (triangle) and $P_{3}$ (circle) schemes.

Figure 2. Divergent FSI $(\Lambda=202)$ simulation of air flow over a rubber wall $\left(\rho_{f} / \rho=1.17 \times 10^{-3}\right)$.

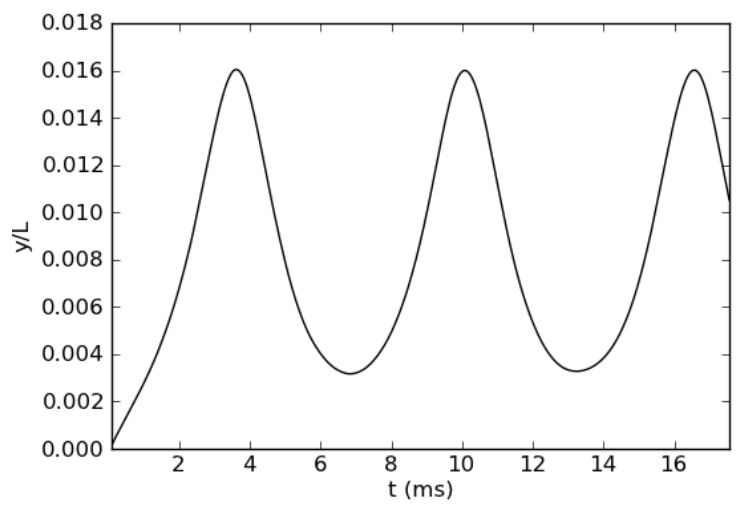

Figure 3. Wall midpoint trace for $P_{3}$ with a divergent FSI $(\Lambda=206)$ simulation of water flow over aluminium $\left(\rho_{f} / \rho=3.7 \times 10^{-1}\right)$.

the fastest, where the wall-time taken to run the simulation on a standard desk-top computer (Intel Q9650 $3.0 \mathrm{GHz}$ processor) was $505 \mathrm{~s}$, based on 50 discretised wall nodes. Schemes $P_{2}$ and $P_{3}$ took longer to run for the same simulation parameters with wall-time taking 1820 s and 1090s respectively. While this may indicate that $P_{1}$ should always be the selected method, this is only for a select set of density ratios of approximately $\rho_{f} / \rho<10^{-2}$. For ratios higher than this, the $P_{1}$ scheme fails to converge due to the inherent numerical stiffness in the problem. By using $P_{3}$ scheme, the case of water flow over aluminium $\left(\rho_{f} / \rho=3.7 \times 10^{-1}\right)$ has been illustrated. This is a case where $P_{1}$ cannot be used and $P_{2}$ has not been used due to its inferior performance.

\section{SUMMARY AND RECOMMENDATIONS}

We now summarise all three schemes and illustrate the different complexity resulting from each coupling method. By taking into account Explicit coupling iterations (E), Newton iterations (N), Krylov iterations (K), GMRES calls (G) and FMM calls (F) we arrive at Table 1. For each case the complexity of G and $\mathrm{F}$ remain constant as they are functions of the wall elements $(n)$. However $\mathrm{E}$ varies vastly $\left(E_{1}>E_{2}\right)$ depending on the conditioning of the left and right hand side, with $\mathrm{K}$ increasing $\left(K_{1}<K_{2}<K_{3}\right)$ for greater functional $(f)$ matrix density and $\mathrm{N}$ increasing $\left(N_{1}<N_{2}<N_{3}\right)$ for greater nonlinearity and reducing accuracy of $\mathrm{K}$.

When using $P_{1}$ we offer no implicit coupling between the fluid and the wall. While Section 4 suggests that this method is the fastest, it cannot be used effectively for all FSI systems. As the magnitude of the unsteady pressure term is governed mainly by the fluid density, not time-step size, the convergence 
Table 1. Number of operations for different coupling $P^{*}$

\begin{tabular}{cccc}
\hline \hline Method & Left eval & Right eval & Total \\
\hline$P_{1}^{*}$ & $\mathrm{E}_{1}\left(\mathrm{~N}_{1}\left(\mathrm{~K}_{1}\right)\right)$ & $\mathrm{E}(\mathrm{G}(\mathrm{F})+2(\mathrm{~F}))$ & $\mathrm{E}_{1}\left(\mathrm{~N}_{1} \mathrm{~K}_{1}+\mathrm{F}(\mathrm{G}+2)\right)$ \\
$P_{2}^{*}$ & $\mathrm{E}_{2}\left(\mathrm{~N}_{2}\left(\mathrm{~K}_{2}(\mathrm{~F})\right)\right)$ & $\mathrm{E}_{2}(\mathrm{G}(\mathrm{F})+3(\mathrm{~F}))$ & $\mathrm{E}_{2}\left(\mathrm{~F}\left(\mathrm{~N}_{2} \mathrm{~K}_{2}+\mathrm{G}+3\right)\right)$ \\
$P_{3}^{*}$ & $\mathrm{~N}_{3}\left(\mathrm{~K}_{3}(\mathrm{G}(\mathrm{F})+2 \mathrm{~F})\right)$ & 0 & $\mathrm{~N}_{3}\left(\mathrm{~K}_{3}(\mathrm{~F}(\mathrm{G}+2))\right)$ \\
\hline
\end{tabular}

behaviour of the $P_{1}$ coupled system is reliant on the fluid-wall density ratio $\left(\rho_{f} / \rho\right)$. When this is relatively high, such as flows involving water or where the ratio is greater than approximately $10^{-2}$, this method cannot converge on a solution due to the numerical stiffness involved with coupling the strong, acceleration sensitive, inertial forces. For low ratios however, the inertial coupling is weak and we can trade simpler NK calculations for small increases in E, with the solution remaining numerically stable.

When higher density ratios are required, $P_{2}$ must be used at a minimum for numerical stability and offers a good trade-off between the cost of the NK and the number of E calculations. This method excels in cases where wall motion remains mildly nonlinear and where inertial forces drive the system dynamics. For cases where E becomes too large, such as for highly nonlinear behaviour or where fluid elements other than potential-flow were used that are vastly more expensive to calculate (such as with the DVM), $P_{3}$ coupling would be preferred. This would be aimed at reducing the number of E critically but would only apply where a full potential-flow coupling offers a reasonable approximation to the real flow.

\section{ACKNOWLedgements}

The main author would like to acknowledge the support of the Australian Postgraduate Awards (APA) and the Curtin Research Scholarships (CRS) schemes.

\section{REFERENCES}

Davies, C. and P. Carpenter (1997). Instabilities in a plane channel flow between compliant walls. Journal of Fluid Mechanics 352, 205-243.

Greengard, L. and V. Rokhlin (1987). A Fast Algorithm for Particle Simulations. Journal of Computational Physics 73, 325-348.

Kapor, J., A. Lucey, and M. Pitman (2010). Fluid-structure interaction using mesh-free modelling. In I. D. . I. H. Kian Teh (Ed.), Proceedings of the 6th Australasian Congress on Applied Mechanics, , pp. 240-249. 12-15 December, Perth, Australia Paper 1041 (eds. Kian Teh, Ian Davies \& Ian Howard).

Katz, J. and A. Plotkin (1991). Low Speed Aerodynamics: From Wing Theory to Panel Methods. McGrawHill.

Knoll, D. and D. Keyes (2004). Jacobian-free Newton-Krylov methods: a survey of approaches and applications. Journal of Computational Physics 193(2), 357-397.

Lucey, A. D., P. W. Carpenter, G. J. Cafolla, and M. Yang (1997). The nonlinear hydroelastic behaviour of flexible walls. Journal of Fluids and Structures 11, 717-744.

Luo, X., Z. Cai, W. Li, and T. Pedley (2008). The cascade structure of linear instability in collapsible channel flows. Journal of Fluid Mechanics 600, 45-76.

Pitman, M. W. (2007). An Investigation of Flow Structure Interactions on a Finite Compliant Surface Using Computational Methods. Ph. D. thesis, Curtin University of Technology.

Trefethen, L. (1996). Finite difference and spectral methods for ordinary and partial differential equations. Cornell University. 